\title{
La prospectiva de los clubes universitarios de ciencia, como estrategia de Responsabilidad Social Universitaria en Tabasco
}

\author{
The prospect of College Science Clubs, as a Social \\ Responsibility strategy in Tabasco
}

Josué Arias Herrera $\otimes$

Universidad Juárez Autónoma de Tabasco

Tabasco, México

Gladys del Carmen Medina Morales

Universidad Juárez Autónoma de Tabasco gladys.medina@ujat.mx

Tabasco, México

$\bowtie$ Autor por correspondencia

\begin{abstract}
Resumen:
Los Clubes Universitarios de Ciencia (CUC) son un conjunto multidisplinario de estudiantes integrados para impartir talleres de ciencia experimentales o demostrativos con el objetivo de acercar al quehacer científico a jóvenes y niños y fomentar su interés por la ciencia. Una estrategia de responsabilidad social, que realiza la Universidad Juárez Autónoma de Tabasco (UJAT) a través de los CUC a partir del 2014. La estrategia consistió en impartición de talleres de divulgación científica a estudiantes de educación media superior del estado de Tabasco. Se presentan datos descriptivos de un caso de estudio sobre su labor de divulgación científica con el propósito de describir la prospectiva de los clubes de ciencia como estrategia de responsabilidad social de la UJAT. El estudio tuvo el objetivo de describir las actividades de la conformación y de desarrollo de los CUC, comprende un periodo de cuatro años en el que se impartieron 140 talleres, en 30 planteles de tres diferentes subsistemas de los 17 municipios del Estado, a 3400 estudiantes con una participación activa de 344 estudiantes como talleristas (M:210, H:134). Con ello la UJAT sensibiliza a un sector importante de la sociedad a interesarse por la actividad científica, en una etapa crucial para decidir su futuro profesional. Este impulso a las vocaciones científicas es un acto primordial de Responsabilidad Social frente a los retos que encara el país.
\end{abstract}

Palabras clave: Responsabilidad Social; divulgación científica; formación de científicos; clubes de ciencia; vocación cientifíca.

Cómo referenciar este artículo:

Arias Herrera, J., \& Medina Morales, G. del C. (2022). La prospectiva de los clubes universitarios de ciencia, como estrategia de Responsabilidad Social Universitaria en Tabasco. Emerging Trends in Education, (4)8A, 1-15. https://doi.org/10.19136/etie.a4n8A.4722

Disponible en:

https://revistas.ujat.mx/index.php/emerging/article/ view/4722

DOI:

https://doi.org/10.19136/etie.a4n8A.4722

Recibido: Aceptado:

$16 / 09 / 2021$ $13 / 11 / 2021$

\section{Publicado:}

05/01/2022

\begin{abstract}
:
The College Science Clubs (CUC) are a multidisciplinary group of integrated students to give experimental or demonstrative science workshops with the aim of bringing young people and children closer to scientific work and promoting their interest in science. A strategy of social responsibility, carried out by the Universidad Juárez Autónoma de Tabasco through the CUC. This strategy began with the clubs in 2014 with the teaching of scientific dissemination workshops to students of upper secondary education in the state of Tabasco. Descriptive data of a case study on his work of scientific dissemination are presented. The study covers a period of four years in which 140 workshops were given, in 30 schools of three different subsystems of the 17 municipalities of the State, to tres mil 400 students. With this, the UJAT sensitizes an important sector of society to take an interest in scientific activity, at a crucial stage in deciding their professional future. This boost to scientific vocations is a primary act of Social Responsibility in the face of the challenges facing the country.
\end{abstract}

Keywords: Social responsability; scientific population; training of scientists; college science clubs; scientific vocation. 


\section{La prospectiva de los clubes universitarios de ciencia, como estrategia de Responsabilidad Social Universitaria en Tabasco.}

\section{| Introducción}

Vivimos en una sociedad red en la que el conocimiento es el valor más importante que se intercambia a través de interconexiones de alcance global (Castells, 1999). Para que las naciones, que aún se encuentran en los espacios periféricos de estos intercambios, puedan asumir un papel más central, deben generar conocimientos científicos y acelerar sus procesos de innovación tecnológica. Esta es una exigencia que plantean constantemente las instancias internacionales como la Organización de las Naciones Unidas para la Educación, la Ciencia y la Cultura (UNESCO, por sus siglas en inglés) y la Organización para la Cooperación y Desarrollo Económico (OCDE.

Desafortunadamente, nuestro país no ha invertido lo suficiente en los últimos años para promover la investigación y la innovación, al menos no en comparación con otras naciones de la región como Brasil. Cifras de la OCDE ubican a México en una posición rezagada a nivel de inversión en investigación, con $0.487 \%$ del Producto Interno Bruto (PIB), cuando la recomendación es del 1\% para países del mismo nivel de desarrollo (OCDE, 2018a). También se presentan rezagos en el número de investigadores per cápita, con 0.817 investigadores por cada mil empleados en 2013 contra la media de la OCDE de 8.108 por cada mil (OCDE, 2018b), y en el número de publicaciones científicas, que llega solo a 90 publicaciones por cada millón de habitantes en 2014 (UNESCO, 2015, p. 18).

Por lo tanto, en México se necesita, de manera urgente, formar nuevos recursos humanos con las competencias genéricas, profesionales y específicas orientadas al desarrollo de estas actividades. Y es que más allá del crecimiento económico que significan la innovación y la investigación para los países, las sociedades en su conjunto se benefician con la divulgación del pensamiento científico, que en tiempos de crisis puede convertirse en una luz en la oscuridad (Sagan, 1995/2017).

Si el ser humano ha vencido en parte las amenazas del hambre, la enfermedad y la guerra, tiene aún frente a sí el reto de la destrucción del planeta, la sobrepoblación y el resurgimiento de los fundamentalismos que nos dividen. Así mismo se hace necesario desvelar los mitos que yacen en torno al quehacer científico (métodos, objetivo, hallazgos y su aplicación) y el impacto, tanto positivo como negativo, que tienen los avances científicos y tecnológicos en la vida de los seres humanos.

La prevalencia del pensamiento mágico sobre
el pensamiento científico. La responsabilidad
social del fomento de una cultura científica.

El poco o nulo interés sobre los temas relacionados con el quehacer científico y tecnológico por parte de la sociedad en general representa una postura peligrosa. Esta falta de interés repercute en generar argumentos con base en datos que carecen del rigor científico necesario, a la toma de decisiones basadas en argumentos carentes de objetividad,cuyosalcances en materia económica, desalud, educación y alimentaria suelen ser de carácter negativo.

En este sentido López (2013), menciona que..."La educación científica es la capacidad de entender temas elementales de ciencia a un nivel suficiente para participar en el debate científico" (párr. 1).

La enseñanza del pensamiento lógico como actividad está orientada a la educación científica, SuárezRodríguez, et al. (2021) menciona que "contribuye a la formación de ciudadanos críticos, tanto en escenarios 
formales como no formales" (párr. 1). Dentro de los clubes universitarios de ciencia se guía a los niños y jóvenes a un proceso formal de investigación, que incluye la exposición y divulgación de resultados.

Así mismo, Namihira afirma que "tener una cultura científica significa que el ciudadano utiliza el conocimiento científico y tecnológico, así como sus resultados para hacer un cambio en su vida como persona y como ente social" (citada por Ruiz Galicia, 2017, párr. 4).

Continuando es esta misma línea Mochán (2020), menciona que en México y otros países de Iberoamérica prevalecen el pensamiento mágico sobre el pensamiento científico, a menos que desarrollemos una cultura científico en donde sea importante establecer el valor de la ciencia y la necesidad del pensamiento crítico, así como señalar el costo de la ignorancia (parr. 5).

El costo de la ignorancia y su impacto pueden ser de gran alcance. En relación a esta podemos mencionar el caso de los detectores moleculares GT200, que fueron adquiridos por el gobierno mexicano entre 2007 y 2010 para localizar cargamentos de droga, armamento y explosivos, aparatos fraudulentos que representaron un riesgo para la seguridad nacional, pues en realidad no detectaban nada. Cada aparato tenía la capacidad de detectar desde una distancia de 300 metros cantidades mínimas de 300 tipos de drogas, además de armas, explosivos, y hasta cadáveres. Para usarlo, los militares debían insertar una tarjeta a la caja negra con la información sobre la sustancia que detectarían y la antena les daría la ubicación de la droga. El misterioso aparato no necesitaba ningún tipo de batería y su vendedor aseguraba que se nutría de la energía electrostática de la persona que lo manipulaba.

Resulta inquietante que aun con la descripción de las cualidades de este "producto milagro" las alarmas no se activaran, sin embargo, los GT200 fueron comercializados en México, Tailandia e Irak. El costo de la ignorancia llego a tenerseriasrepercusiones, talcomoserelataenlagaceta del senado de la república mexicana del 13 de febrero de 2014.

A pesar de que desde el ámbito científico hubo alertas acerca de la ineficacia y fraude que representaron estos equipos de "seguridad", el gobierno federal adquirió un total de 1, 112 equipos, lo que represento un total de 450 millones de pesos (Monreal Avila, 2014, párr. 3).

A lo anterior se suman las múltiples arbitrariedades cometidas en lo que concierne a derechos humanos, que fueron desde detenciones hasta cateos ilegales por parte de militares y cuerpos policiacos; se estima que aproximadamente 5,000 personas habrían ido a la cárcel y habría ocasionado más de 1,000 cateos ilegales, motivados en que el "dispositivo" apuntaba a domicilios que supuestamente tenían droga o armas en su interior. Para 2015, 1,980 personas seguían detenidas por causa de las "detecciones" de los GT200.

Frente a este claro ejemplo de fraude, en México se han hecho llamados al Gobierno Federal, por un lado, para que retire estos detectores moleculares de los retenes de donde se utilizan; incluso la Comisión Nacional de los Derechos Humanos, en el año 2011 hizo una recomendación a las fuerzas armadas respecto a los cateos que realizaban para justificar las detenciones, a pesar de que el gobierno de Gran Bretaña advirtió de la poca eficiencia del detector (Monreal Avila, 2014, párr. 6).

En este escenario, la luz de la ciencia puede cambiar la vida de los jóvenes y darles las herramientas para forjarse un futuro más acorde a las demandas del mundo globalizado en el que vivimos. Por lo que inducir el interés por la investigación, el conocimiento científico, la ciencia y la innovación entre las nuevas generaciones, es tarea impostergable, al mismo tiempo, un acto de responsabilidad social que asumen organismos públicos 
y privados, como la universidad, que tengan plena conciencia de los retos a los que nos enfrentamos como sociedad. La difusión de la ciencia, es uno de los caminos naturales para ejercer su responsabilidad social.

\section{La investigación como responsabilidad social universitaria}

En la literatura sobre Responsabilidad Social Universitaria (RSU) en Latinoamérica, la investigación es concebida como uno de los pilares del quehacer universitario, y se le identifica como un área en la cual la universidad puede ayudar a resolver los problemas de la sociedad a la que pertenece. Así, se enfatiza que la investigación realizada debe tener pertinencia y utilidad social, involucrando a actores de la comunidad (Vallaeys, De la Cruz y Sasia, 2009), que se debe mantener el equilibrio entre investigación básica y aplicada (Remolina, 2003), y que las líneas de investigación deben contribuir al desarrollo del territorio (Gaete Quezada, 2015), pues "una universidad socialmente responsable significa por definición una universidad anclada en su territorio" (Vallaeys, 2014, p. 5).

La universidad, no solo debe producir conocimientos científicos, también debe difundirlo a la sociedad en su conjunto, pues como propuso la UNESCO (2009) en su Conferencia Mundial sobre la Educación Superior:

[...] ante la complejidad de los desafíos mundiales, presentes y futuros, la educación superior tiene la responsabilidad social de hacer avanzar nuestra comprensión de problemas polifacéticos con dimensiones sociales, económicas, científicas y culturales, así como nuestra capacidad de hacerles frente (p. 2).

Sobre la expresión RSU, Gaete Quezada (2015), señala que:

el concepto (...) es originario del ámbito empresarial, sus raíces más importantes se encuentran en la filantropía y el altruismo, que ha evolucionado hacia planteamientos relacionados con la ética, la rendición de cuentas y la sostenibilidad como sus principales formas de expresión entre las organizaciones empresariales (pp. 3-4).

Para Ostrom (1990), la RSU “es la regulación ética y política responsabledelosprocesos desencadenadosporlasociedad tecno-científica". Para Jonas (1979), nuestra sostenibilidad planetaria depende de la responsabilidad para con nuestra propia evolución, por consiguiente, el movimiento de la 'responsabilidad social de las organizaciones', se inscribe en esta lógica ética y política de regulación.

En el ámbito universitario, François Vallaeys (2014, p.107) señala que la responsabilidad social, "Es la gestión justa y sostenible de los impactos universitarios". La Universidad, según Vallaeys (s/f), es:

Un lugar estratégico para instituir y promover la responsabilidad social de la ciencia es justamente la Universidad, porque es el lugar de convergencia entre la producción del saber científico (la investigación), la reproducción de este saber (transmisión de los conocimientos y formación de los ciudadanos profesionales que socializan las ciencias $y$ tecnologías) y la información del gran público sobre el quehacer científico (la Universidad es un espacio social abierto al debate público) (pp.13-14).

Esta labor de divulgación científica la Universidad Juárez Autónoma de Tabasco la ha asumido como parte de su RSU, difundiendo los resultados de la investigación que realiza a través de muy diversos canales tanto académicos como de alcance masivo (por ejemplo, la radio y televisión universitarias).

Así mismo, ante la problemática que tiene la región, por la escasez de recursos humanos calificados, la UJAT realiza diversas estrategias de cobertura intra e interinstitucional para promover las vocaciones científicas, con eventos 
como el Verano de la Investigación Científica y el Encuentro de Jóvenes hacia la Investigación, que se llevan a cabo anualmente, así como el proyecto que hace énfasis en la divulgación científica, denominado "Club de Ciencias Universitarios (CCU), que destaca por su alcance, al incluir a los estudiantes de Educación Media Superior del Estado de Tabasco, y que es el caso de este estudio.

\section{Los Clubes de Ciencia como un caso de éxito de responsabilidad social universitaria}

Toda profesión requiere de determinadas competencias para que el desempeño profesional sea exitoso. Por ello, un estudiante talentoso en ciencias debe acercarse lo más posible a un perfil idóneo: curioso, observador, metódico y analítico. Este perfil responde a las actividades propias de un científico, ya que su desempeño tendrá como base la capacidad de plantear preguntas, establecer métodos de mediciónygenerarrespuestassobreelfenómenodeestudio.

De acuerdo con la UNESCO (1984), los Clubes de Ciencia (CC), son una asociación permanente de jóvenes con una organización establecida que, orientados por asesores debidamente calificados, desarrollan actividades que contribuyen a la educación científica y tecnológica de sus miembros.

A nivel internacional, países como España y Argentina han incluido en sus agendas educativas la figura de los Clubes de Ciencia con el objetivo de propiciar espacios de fomento a lasactividades escolares decaráctercientíficoy tecnológico.

Diaz y Rojas (2020) señalan a los clubes de ciencia como esos espacios para estudiante que buscan el desarrollo habilidades que dan respuesta a su curiosidad, abriendo la alternativa de compartir experiencias que den sentido de pertenencia y, contribuyendo a un proceso de aprendizaje.

En México, el Consejo Nacional de Ciencia y Tecnología (CONACYT) se ha ocupado de generar programas que abonen a la ciencia, debido a ello ha asignado techo financiero a diversos programas que fomentan el desarrollo de la investigación, de esta forma surge el Programa de Apoyo para el Fomento de las Vocaciones Científicas y Tecnológicas en Jóvenes Mexicanos, un instrumento que busca poner en contacto directo a la ciencia y tecnología con los jóvenes, a través de prácticas y actividades coordinadas y desarrolladas preferentemente por científicos y académicos a nivel nacional, mismo que apoya cinco líneas generales, entre las que destaca: apoyo para la realización de cursos que fomenten vocaciones científicas y tecnológicas en jóvenes y niños de manera permanente y sistemática.

Dado que no existe un programa federal específico, las iniciativas institucionales locales se han creado de acuerdo a necesidades e intereses específicos. En ese tenor, a inicios del 2014, se conformó una fundación bajo la denominación de Clubes de Ciencia en México (CdeCMx), que es una organización sin fines de lucro creada por jóvenes científicos mexicanos y estadounidenses, conformados para promover las oportunidades profesionales en las áreas de la ciencia y la tecnología.

Cabe hacer mención que uno de los objetivos de los clubes de ciencia en la de divulgar ciencia. En la actualidad, gracias al trabajo de muchos apasionados de esta noble labor, ésta ha pasado de ser un simple pasatiempo entre los apasionados del área del conocimiento a la que pertenecen (física, química, ingeniería, biología, medicina, etc.), a una práctica profesional, en donde los métodos, objetivos y formatos son diversos $\mathrm{y}$ cuentan con lineamientos establecidos para su praxis.

De forma general, la divulgación científica se define como:

El conjunto de actividades de promoción y circulación del conocimiento construido por medio de métodos científicos, los cuales tienes una importancia en el contexto social, cuyo propósito 
es hacer que el conocimiento científico sea accesible a toda la sociedad, lo que debería incidir positivamente en el desenvolvimiento de una determinada civilización, así mismo, es una labor multidisciplinaria, cuyo objetivo es comunicar el conocimiento científico, utilizando diversos medios, a diversos públicos voluntarios recreando este conocimiento con fidelidad y contextualizándolo para hacerlo accesible (Lozada-Chávez, 2006, párr.5).

Los formatos en los en que se desarrolla son diversos, que van desde talleres, obras de teatro, videos, publicaciones en periódicos y revistas, así como los clubes de ciencias. Estos últimos integran una serie de actividades, en la que podemos destacar las denominadas como lúdicas o recreativas que van enfocadas a la creación de una experiencia científica, mediante la generación de eventos agradables realizando actividades científicas; se busca re-crear las experiencias científicas, con la particularidad, de que se emplean materiales que suelen ser fácilmente asequibles. En este sentido, se prima la experiencia de los sujetos que participan en la planeación y desarrollo de las actividades de divulgación que pertenecen, lo que se denomina como recreativa o lúdica.

En primera instancia tenemos a quien expone (tallerista, ponente,guía), quieneselencargo deadaptarlos contenidos (física, química, biología...) a un lenguaje más ameno para el público objetivo. Esto supone un mayor razonamiento de por parte de quien pretende (alumno, practicante, maestro) explicar determinado fenómeno (físico, químico, social); estando en la necesidad de consultar fuentes, ampliar la investigación sobre algún tema en específico o buscar mentoría con el o los expertos en la materia de determinado campo. Todo lo anterior lleva, a quien expone, a profundizar en lo que pretende explicar y ampliar la perspectiva del cómo abordar el fenómeno a divulgar.

En segunda instancia, el público objetivo (estudiantes, maestros, niños, padres de familia), quien tiene la oportunidad de ser parte de la recreación de un evento científico, ya no son solo un espectador o un mero receptor pasivo, sino como el protagonista de cada uno de los momentos. A través de los experimentos, dinámicas, representaciones o juego, irá descubriendo por sí mismo la significancia de los conceptos que se pretende que aprenda, activando la capacidad de asombro, estimulando el pensamiento crítico y sobre todo, cambiando la percepción de que la ciencia es solo para personas superdotadas, que es aburrida e irrelevante.

Enla actualidad existengruposyasociaciones, en diferentes partes de la república mexicana, que se dedican a la divulgación científica con el enfoque de ciencia recreativa, ciencia lúdica o ciencia divertida. Algunos realizan esta labor de manera independiente, en otros casos acceden a financiamientos públicos o privado o pertenecen a alguna universidad o institución educativa. Sus aportaciones van desde implementación de jornadas, municipales, estatales o naciones de divulgación, la realización de jornadas, coloquios y congresos en los que se discuten nuevos planteamientos con relación a la investigación y experiencias producto de la labor divulgativa. Por nombrar algunas, está la Sociedad Mexicana para la Divulgación de la Ciencia y la Tecnología (SOMEDICYT) cuya misión es:

Contribuir al fortalecimiento de la cultura científica de la población, a través de una divulgación profesional, efectiva y pertinente, para fomentar la apropiación social de la ciencia y tecnología; la comprensión del mundo desde una perspectiva científica; la toma de decisiones; y el bienestar personal y colectivo (SOMEDICYT, 2019, párr. 2).

De igual manera la Recreación en Cadena, Red Mexicana de Talleristas de Ciencia, quienes por medio de:

Los talleres de ciencia recreativa buscan que las personas interactúen con la ciencia y la tecnología (C y T) de forma completa: a niveles físico, intelectual y 
emocional, y que las convierte en protagonistas en el proceso de divulgación científica. Son procesos lúdicos en que los participantes asumen el rol de investigadores para conocer la historia, forma de trabajo, resultados y hasta retos pendientes de la Cy T (Red Mexicana de Talleristas de Ciencia, 2021, párr. 1).

Por ello, en el 2012 la Universidad Juárez Autónoma de Tabasco (2012) se planteó el Plan Institucional de Desarrollo de la Investigación dentro del Eje 3. Fomento a la Investigación, la creación de Clubes Universitarios de Ciencia (CUC) con el objetivo general de impulsar, promover y divulgar el quehacer científico entre los estudiantes en el nivel medio y medio superior y de manera específica, formar a los estudiantes-talleristas en divulgación científica, diseñar e impartir los talleres de divulgación y evaluar el impacto de cursos informarles en la definición de la vocación científica de los alumnos que cursa el nivel medio superior.

En tal contexto, en el 2014 surge el interés de crear un proyecto financiado por el CONACYT que arropara el establecimiento y seguimiento de los clubes universitarios de ciencia, como un trampolín no formal en el que convergen jóvenes estudiantes, talleristas, profesores y adultos en general para exponer ideas, leyes, eventos científicos, naturales o sociales de manera ingeniosa y de esta manera fomentar dos cosas; uno, la alfabetización científica y dos, el gusto por la ciencia ya que una de las causas del bajo interés por hacer ciencia es el desconocimiento de quehacer científico y su potencialidad en el desarrollo profesional. Una alternativa para contrarrestar esta mala percepción en los estudiantes, era crear programas de orientación vocacional y científica que busca proporcionar al adolescente (en vías de elegir su profesión), un panorama sobre los alcances de la investigación y su impacto en el desarrollo socioeconómico de un estado o nación. Asimismo, se consideró realizar actividades científicas prácticas e interactivas que logren despertar el interés en el mundo de la ciencia a través de la divulgación científica y tecnológica.

Bajo esta mirada de la divulgación, en años recientes, el Consejo de Ciencia y Tecnología en el estado de Tabasco creó el programa de Ciencia en Movimiento, el cual, en esencia, persigue la misma filosofía: hacer difusión de la ciencia, habilitar a estudiantes en divulgación científica, entre otras cosas.

En la UJAT, actualmente hay grupos de estudiantes en las áreas de ciencias ambientales, ingeniería química e ingeniería en acuacultura, que incluyen entre sus actividades la impartición de pláticas relacionadas con sus respectivas profesiones, a estudiantes de menores niveles educativos. Aunque los resultados de las actividades que han realizado estos grupos de estudiantes eran alentadores, no habían obtenido una capacitación y habilitación formal y metodológica en la divulgación científica, que promoviera e impulsara en los estudiantes del nivel medio superior el interés de considerar las profesiones científicas como forma de vida laboral.

El proyecto se inició con la conformación de 7 CUC's, integrados por estudiantes del área de Ingeniería y Arquitectura, Ciencias Biológicas, Ciencias Sociales y Humanidades, Educación y Artes y una División Multidisciplinaria. Cabe destacar que la mayoría de estos estudiantes ya realizaban la Divulgación Científica sin un reconocimiento institucional. De modo que el objetivo del estudio es describir y explicar la prospectiva de los clubes universitarios de ciencia como una estrategia de responsabilidad social universitaria de la UJAT ya que como menciona Max Scheler (1979), se debe posibilitar el proceso formativo (...) de quien forma, eliminar los obstáculos internos y externos, convertir el impulso de adquisición de una formación en una voluntad de formación inteligente y consciente de sus fines, y dirigida hacia un contenido valioso. En este sentido, los integrantes en cuatro años de trabajo continuo, los integrantes de los CUC se han hecho acreedores a la 
formación en divulgación científica con expertos en el oficio y de reconocido prestigio nacional e internacional.

\section{| Método}

Se presenta un estudio cuantitativo descriptivo explicativo para el cual se consideró un periodo de cuatro años de información recabada. El estudio tuvo el objetivo de describir las actividades de la conformación y de desarrollo de los CUC en la UJAT.

La UJAT es una universidad pública que cuenta con 33 mil alumnos aproximadamente, distribuidos entre doce divisiones académicas y un centro de lenguas que la conforman. Se presenta un estudio cuantitativo descriptivo que se desarrolló con un muestreo polietápico, con sujetos voluntarios.

Se visitaron 30 planteles de tres diferentes subsistemas de educación media y media superior de los 17 municipios que conforman el estado de Tabasco. Se contó con la participación de 344 estudiantes de distintas licenciaturas de la institución que fungieron como talleristas, de los cuales 210 fueron mujeres y 134 hombres.

Se hace énfasis en que los datos que se presentan son sobre la conformación y desarrollo de experiencias y experimentos de los Clubes de ciencia de la UJAT. La muestra fue conformada por siete CUC integrados por alumnos de las Divisiones Académicas de Ciencias Básicas, Ingeniería, Educación y Artes, Ciencias Biológicas y Ciencias Sociales y Humanidades. Tres de ellos fueron de las divisiones académicas multidisciplinarias.

La Secretaría de Investigación, Posgrado y Vinculación elaboró un cuestionario que constó solo de dos apartados, la primera sección con información sociodemográfica, y la segunda, con preguntas relacionadas al taller experimental de mayor interés. Se hace énfasis que se documenta el proceso como un caso de buena práctica en relación a la responsabilidad social universitaria. Para el procesamiento de los datos, se elaboró una hoja de cálculo en donde se capturó cada uno de los cuestionarios aplicados.

\section{| Resultados}

Como se describió en el apartado anterior, el objetivo del estudio fue uno de los primeros pasos que se dieron fue la creación de una imagen, para que el estudiante se sintiera identificado y reconocido en la tarea que realizaba.

Es importante mencionar que cada uno de los CUC presentó una propuesta, sin embargo, en su mayoría se orientaba a imágenes del área de las ciencias exactas; se requería un logo que fuese inclusivo para todas las disciplinas. Después de varias pruebas, se optó para considerar la figura 1 como el logo institucional de los CUC, un logo que conlleva la regionalidad (con la jícara) y que a su vez incluye las siglas de cada una de las Divisiones Académicas que cuentan con Clubes de Ciencia.

Figura 1

Logo de los CUC's de la UJAT

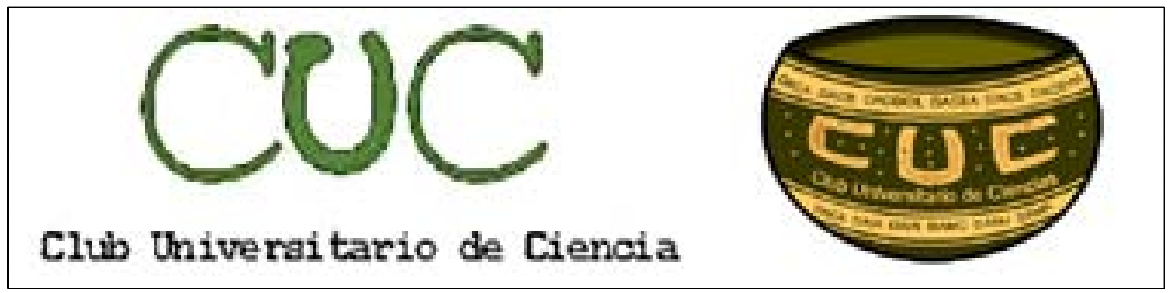


Se proveyó a los estudiantes de materiales académicos

especializados para realizar las actividades.

y químicos, así como de instrumentos y equipos

\section{Figura 2}

CUC's-UJAT realizando actividades con estudiantes de otros niveles educativos

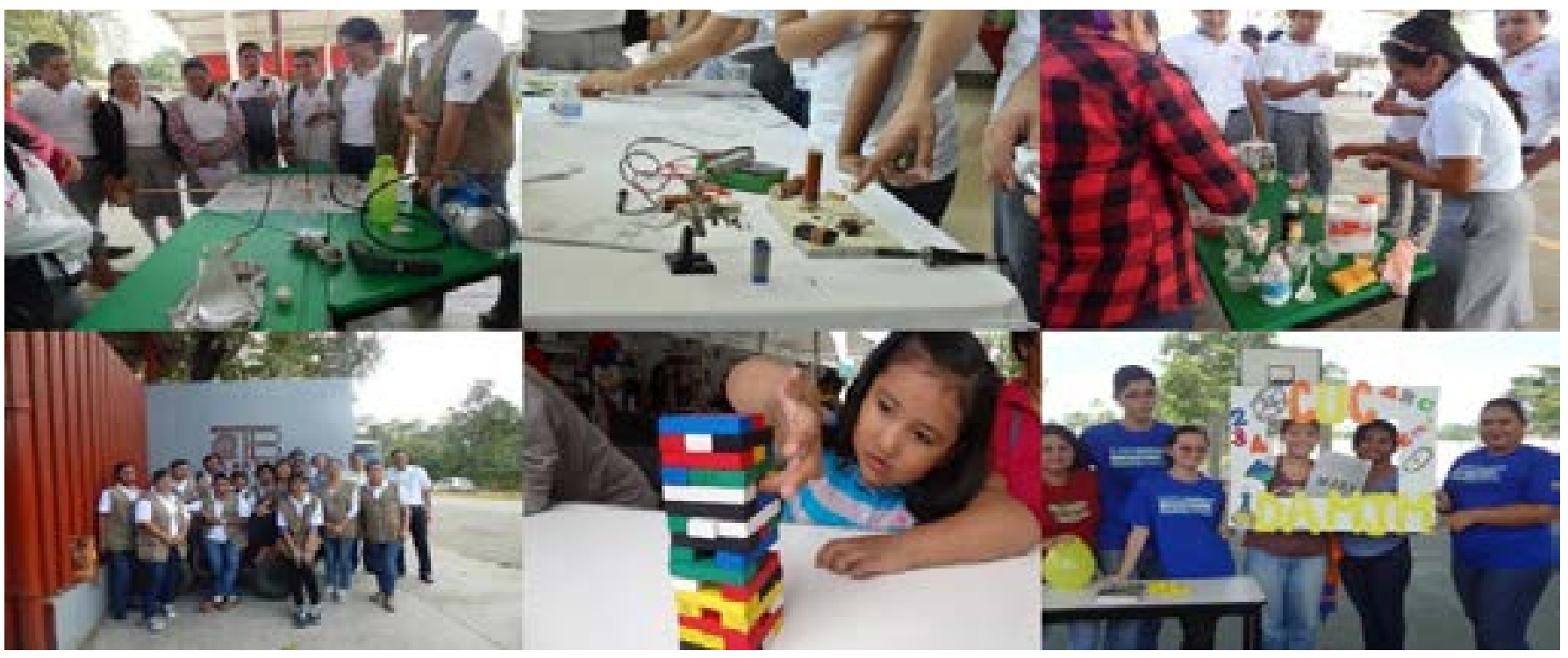

En septiembre de 2014 se creó un grupo en Facebook para poder administrar las actividades, atender sugerencias, comentarios y difundir las actividades que los estudiantes iban a realizar.

Figura 3

Página de Facebook de los CUC's de la UJAT.

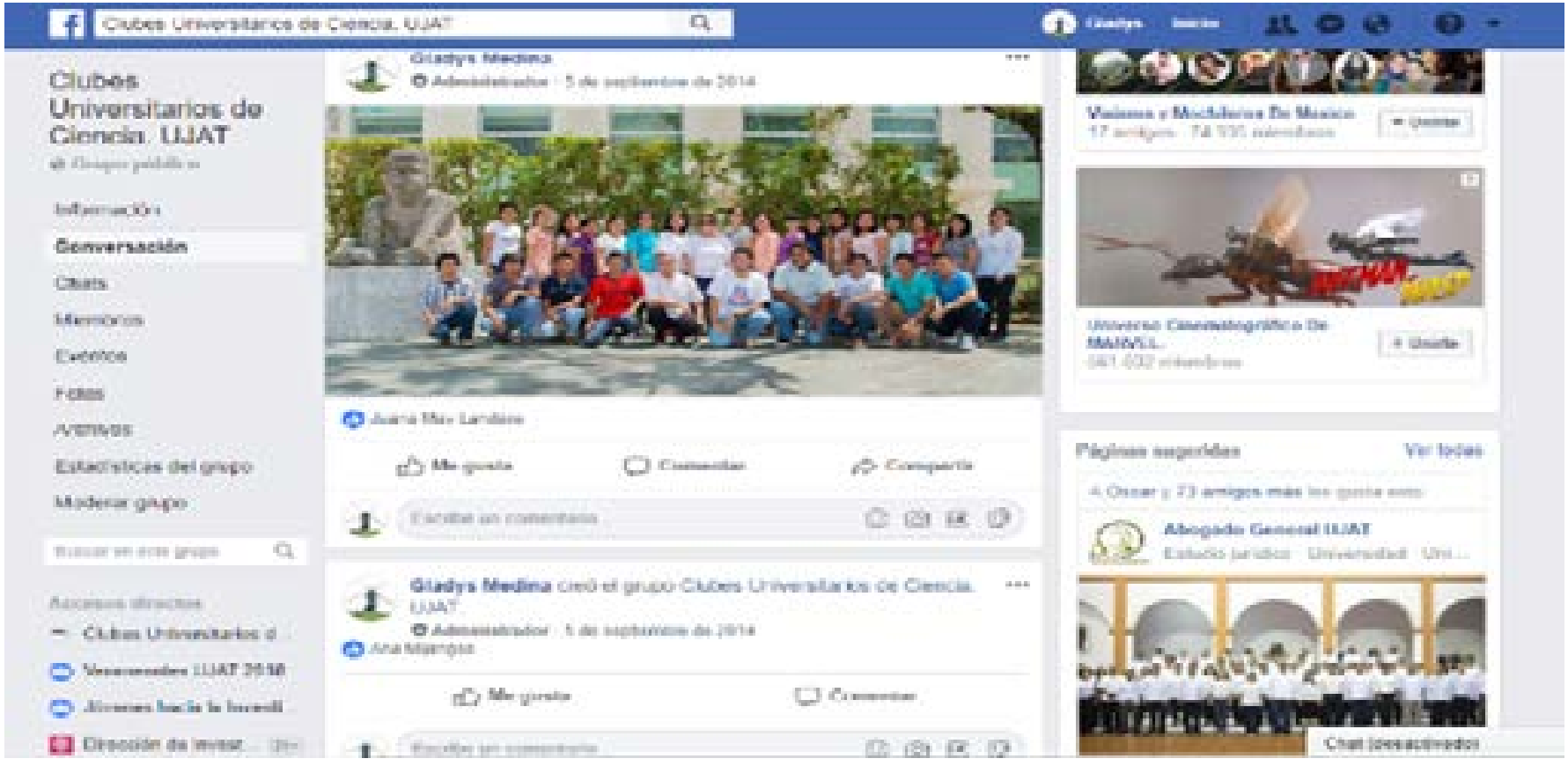

Los CUC diseñaron 148 talleres para impartir en las visitas a cada uno de los colegios de bachilleres de todos los municipios desde Huimanguillo hasta Tenosique, y en total participaron 3400 estudiantes de los niveles de tercero, cuarto y quinto semestre de preparatoria de los cuales 1890 fueron encuestados. Se visitaron los 17 municipios del Estado, y se cubrieron tres subsistemas de Educación Media Superior (Colegios de Bachilleres, Colegio 
Nacional de Educación Profesional Técnica [CONALEP] y Colegios de Estudios Científicos y Tecnológicos [CECYTE]).

Hubo una participación de 324 estudiantes de las Divisiones Académicas ya mencionadas, que diseñaron y/o impartieron los talleres de Divulgación Científica, mismos que se dieron a la tarea de planificar las actividades considerando la audiencia a quienes fue dirigido, esto significa que dichos talleres se replicaron 1608 veces en total. Esta tarea, Gaeta Quezada (2015) lo define como "aprendizaje servicio que permite a los estudiantes, relacionar su formación profesional con la búsqueda de soluciones concretas a las problemáticas sociales, desarrollando un mayor compromiso con los intereses de la sociedad y no únicamente con los aspectos propios de su perfil profesional" (p.101).

El impacto de los talleres impartidos puede visualizarse en la siguiente figura en la que se presentan los talleres más gustados, hay que recordar que los talleres se replicaron en función de la audiencia, disponibilidad de materiales y expertise del tallerista.

\section{Figura 4}

Talleres más gustados.

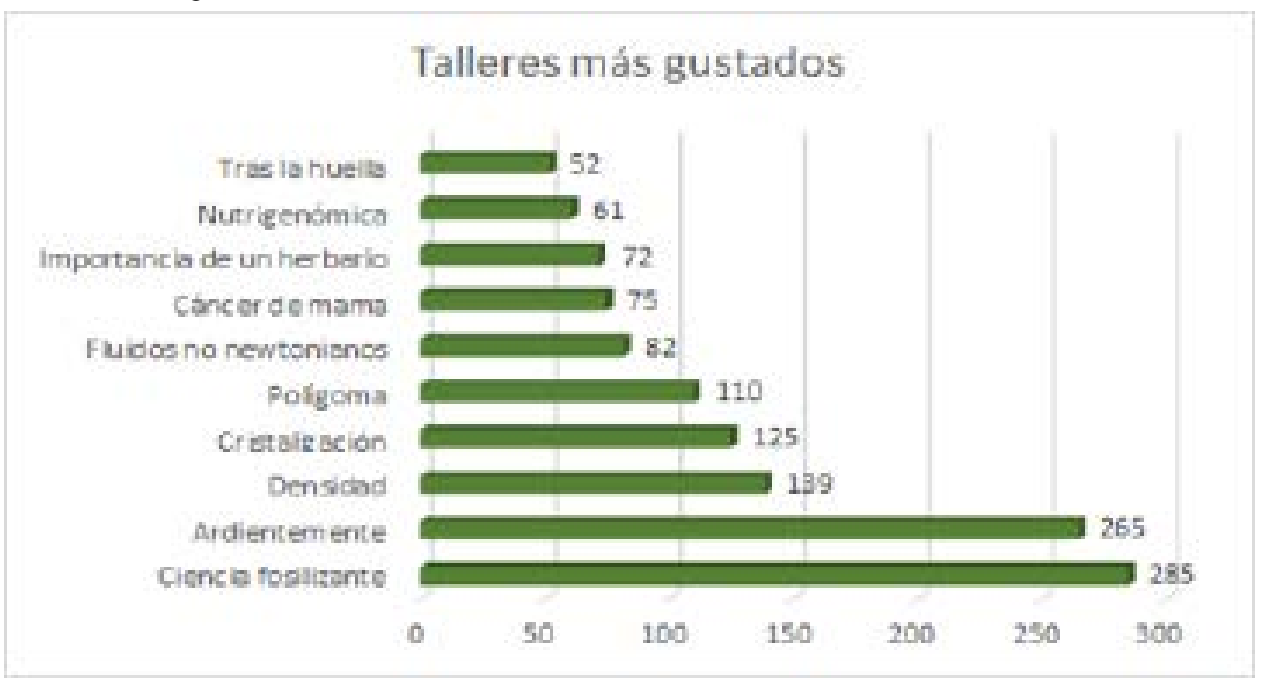

La participación de las mujeres en actividades académicas, científicas o de divulgación se ha incrementado de manera significativa en la UJAT, al menos, eso reflejan las estadísticas del programa del Verano de la Investigación
Científica y el Encuentro de Jóvenes hacia la Investigación. La figura 5 muestra la participación de más del 60\% de las mujeres en actividades de los CUC.

Figura 5

Participación por Género en actividades de los CUC's de la UJAT

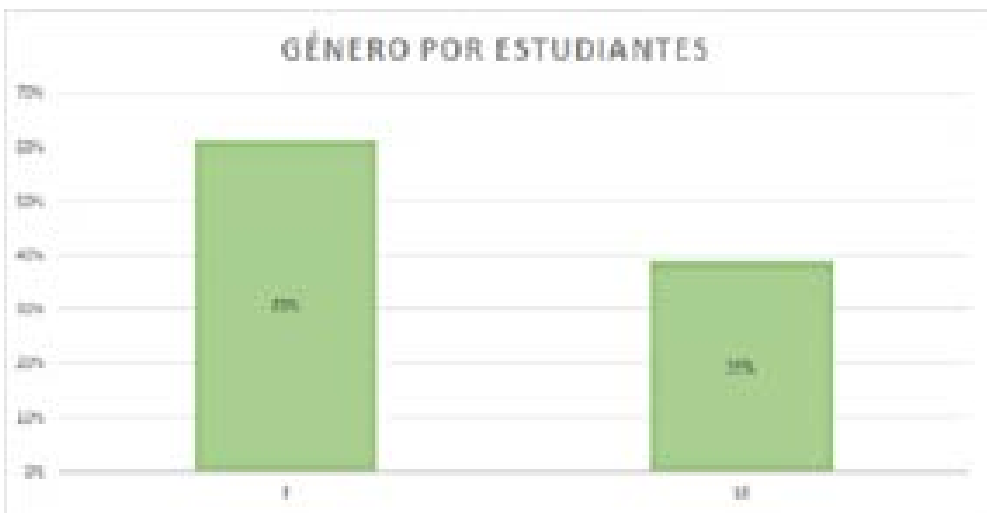


El CUC de la División Académica de Ciencias Sociales

y Humanidades, elaboró un Reglamento para la participación en actividades académicas y científica de los CUC; esto con la finalidad de establecer políticas y criterios para la aceptación de integrantes, consiguiendo con ello, una participación comprometida.

En el marco del 3er Encuentro de Jóvenes hacia la Investigación en el 2015, se realizó la 1ra. Feria de Clubes Universitarios de Ciencia en el que se presentaron talleres a estudiantes de escuelas invitadas, así como a la comunidad universitaria de otras Instituciones que participaron en el Encuentro. Esto ha servido como plataforma para generar interés en buscar fuentes de financiamiento para crear estos Clubes en sus dependencias. Tal como se visualiza en las figuras $6 a$ y 6 b.

Se realizaron en total nueve Ferias de Ciencia en las Divisiones Académicas con el objetivo de dar a conocer las actividades que los estudiantes realizaron en distintos escenarios y que a su vez generara una inercia de trabajo, un interés por parte de los pares para unirse a dichas actividades. En la división de Educación y Artes se contó con una participación aproximada de 150 estudiantes, como se muestra en las figuras siguientes:

\section{Figura 6a}

Primera Feria de los Clubes Universitarios de ciencia.

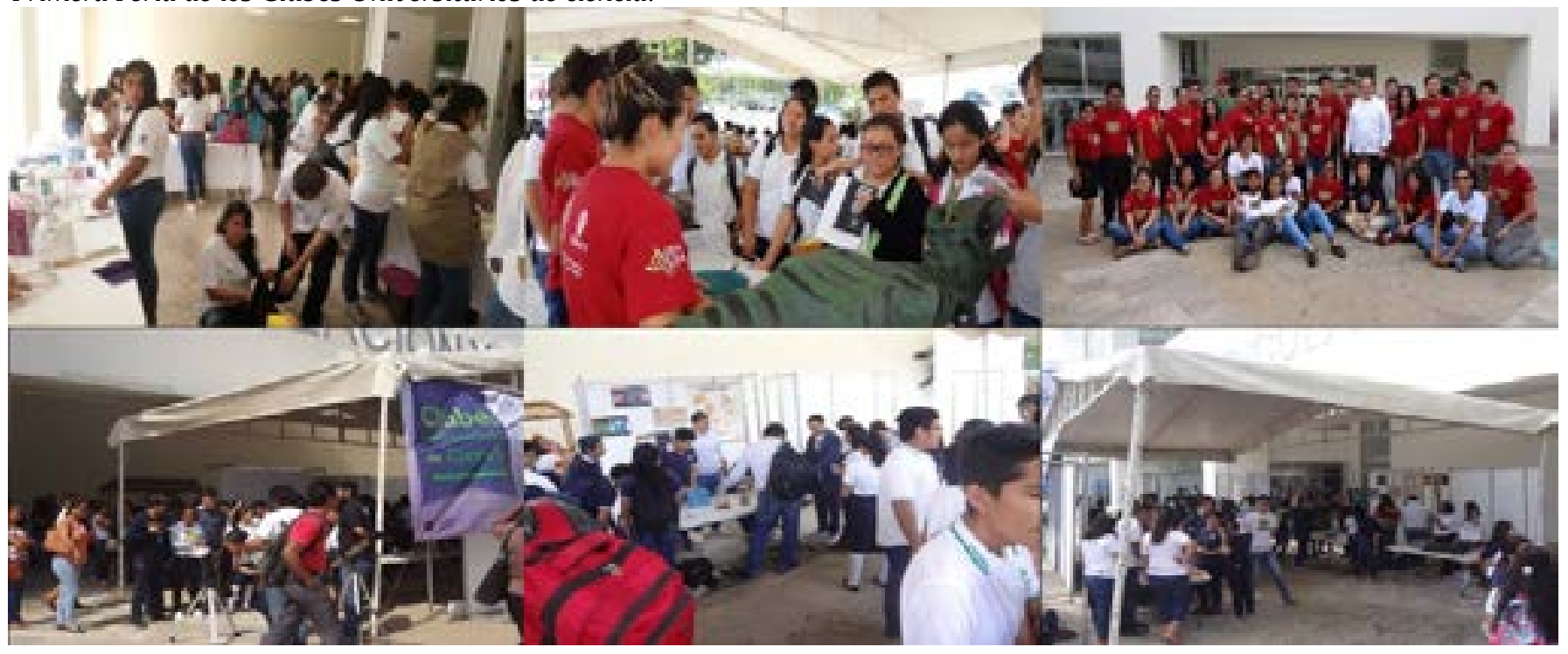

Figura $6 \mathrm{~b}$

Primera Feria de los Clubes Universitarios de ciencia

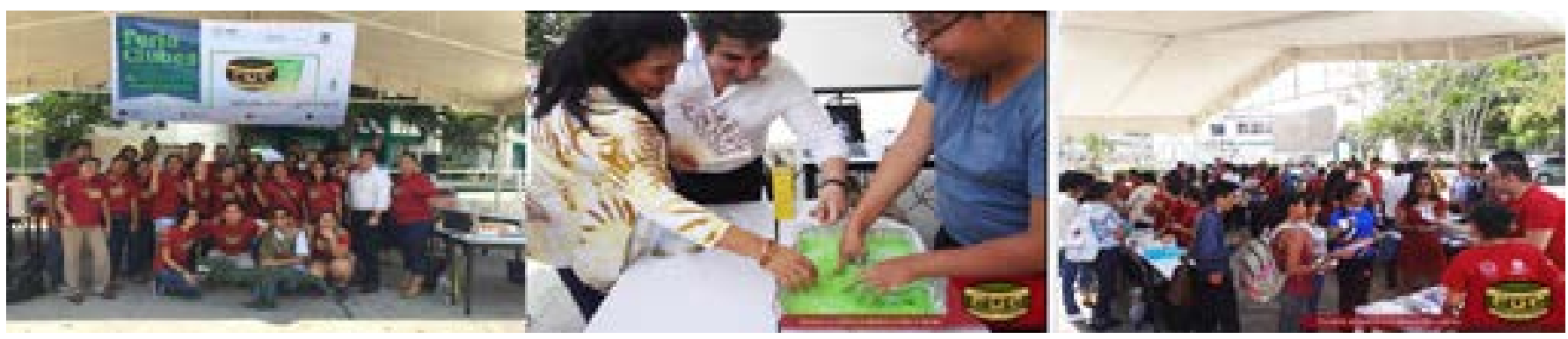

Tal como se presenta en la figura 7 , se publicó una convocatoria institucional para que estudiantes, prestadores de servicio social y/o prácticas profesionales participaran en los CUC. Contando con la participación de 28 estudiantes de Ingeniería, Ciencias Básicas, Sociología, Historia, Comunicación, Idiomas y Ciencias de la Educación. Así como la participación en Cursos de Inducción. Esta iniciativa se consideró a partir del interés de los estudiantes por participar en actividades de divulgación científica. Entre las tareas principales estaban las de diseñar talleres y acudir a instancias educativas a impartirlos. 


\section{Figura 7}

Prestadores de Servicio social y Prácticas Profesionales participantes en los CUC's

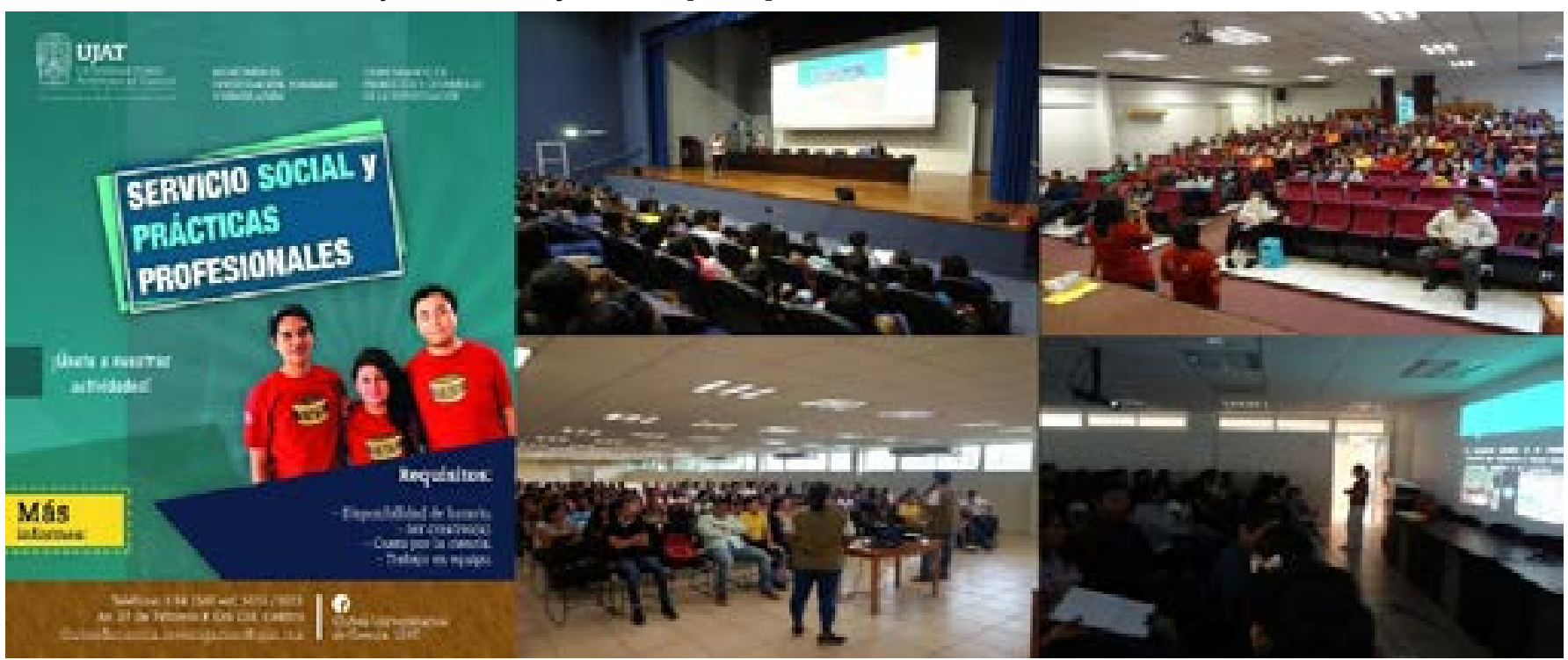

La figura 8 muestra cinco de las conferencias impartidas sobre divulgación de la ciencia con el objetivo de fomentar el gusto a la divulgación científica entre los estudiantes, así como para propiciar el trabajo colaborativo. Podemos destacar la participación de Ángela Swafford, Estrella Burgos y Cecilia Rosen, todas ellas, destacadas periodistas de la ciencia.

\section{Figura 8}

Conferencistas en eventos de los CUC's

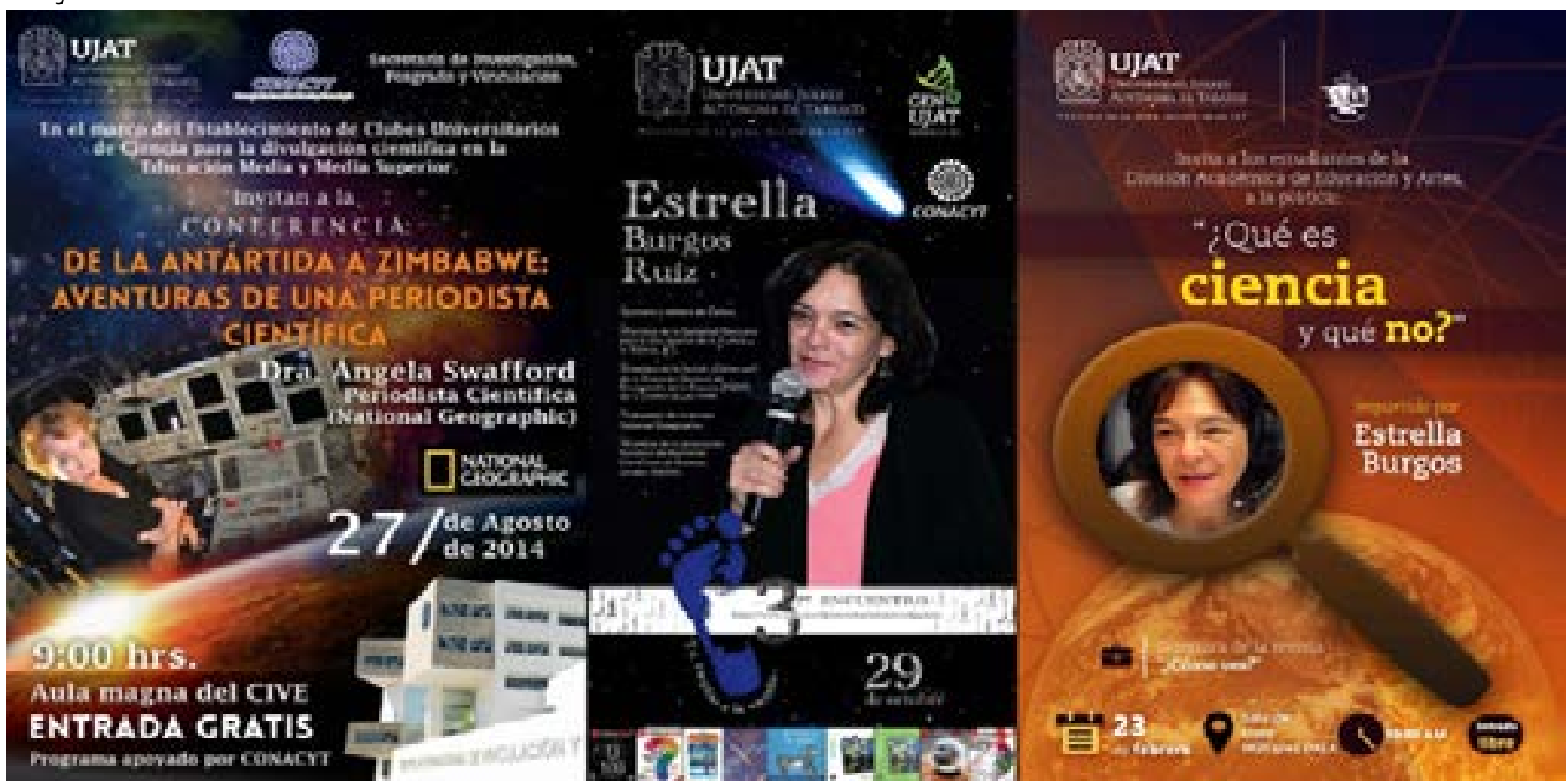


La figura 9 muestra algunas de las sesiones de los ocho talleres que recibieron los cuc's, en relación a la divulgación científica, para en primer lugar, abonar a la formación de los estudiantes talleristas y dos, para reconocer su tarea; esto se vio reflejado en la generación de talleres más didácticos y sistematizados, que se lograron compilar en el Manual del Divulgador.

\section{Figura 9}

Talleres sobre la Escritura en la divulgación científica

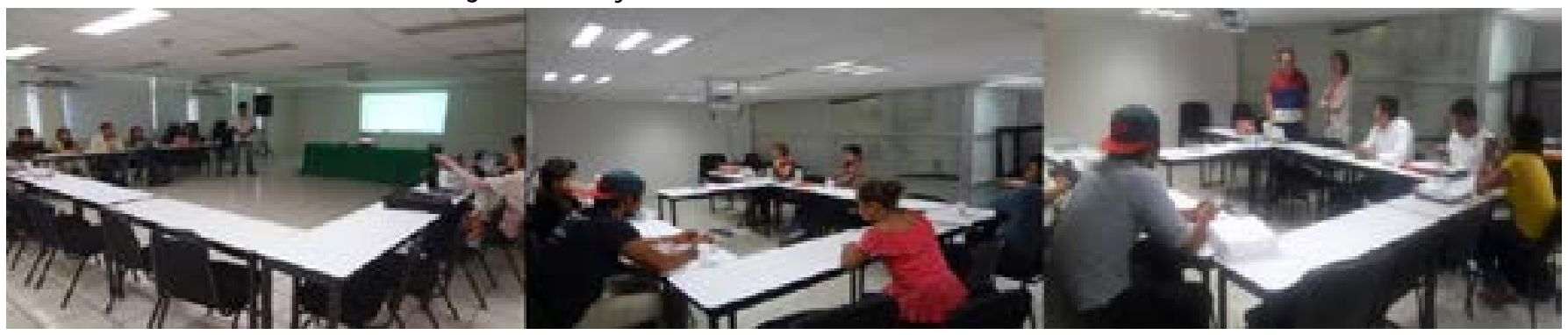

El Manual del Divulgador compila un total de 17 Talleres sobre cómo divulgar la ciencia. Su estructura se conforma de los siguientes apartados: Área de conocimiento, Título- Asunto, ¿Qué buscamos? ¿́ ¿De qué trata?, ¿Qué es esto?, ¿Qué necesitamos? ¿Dónde lo hacemos?, ¿Para quién es? ¿Para cuántos?, Tiempos, ¿Cómo se hace? Manos a la obra, ¿Qué pasó y por qué?, 'Un poquito más, Mucho ojo. ¡Aguas! y Glosario de términos. Este Manual se encuentra en Revisión Editorial para su trámite de ISBN.

\section{| Conclusiones}

Se describió un caso de éxito de Responsabilidad Social Universitaria, el cual brinda la oportunidad de observar experiencias ubicadas en un ámbito geográfico específico y en un contexto particular, que tienen en ellas la semilla para replicación en otros ámbitos. De ahí su utilidad. Yin (1989), establece que una investigación empírica que investiga un fenómeno contemporáneo en su contexto real, donde los límites entre el fenómeno y el contexto no se muestran de forma precisa, y en él, múltiples fuentes de evidencia son utilizadas. Tomando esto como referencia la UJAT, a través de los CUC sensibiliza a un sector importante de la sociedad a interesarse por la actividad científica, en una etapa crucial para decidir su futuro profesional. Este impulso a las vocaciones científicas es un acto primordial de Responsabilidad
Social frente a los retos que encara el país.

Como puede verse en los resultados, los CUC de la UJAT han generado un impacto significativo en la percepción y formación de los universitarios respecto al quehacer científico y su divulgación. Con las actividades realizadas por los miembros de los CUC, así como los usuarios y beneficiarios directos de los Planteles de Educación Media y Media Superior en Tabasco, que tomaron parte en las actividades, se han fomentado las vocaciones científicas y el interés por hacer y divulgar la ciencia.

Es probable que estos estudiantes tendrán una visión y posición de mayor alcance frente a los desafíos de la sociedad global y de los retos ambientales, demográficos y sociales que, imponen, a todos, la urgencia de promover la ciencia y la técnica para preservar el bienestar de la especie humana en el presente siglo. Sobre todo, que, al divulgar la ciencia a nuevas generaciones, desarrollan su propia responsabilidad social universitaria. La difusión del quehacer científico entre las nuevas generaciones es un claro acto de responsabilidad social, que trae beneficios inmediatos a los jóvenes bachilleres, ya que algunos así pueden ampliar sus horizontes respecto a las opciones profesionales existentes, yenelmejor delos casos descubrir su vocación por la ciencia al momento de elegir carrera. 
La investigación, que es una de las tareas fundamentales de la universidad, le brinda además multitud de oportunidades para que ésta cumpla su potencial de institución socialmente responsable. En este trabajo se presentó un caso de éxito en el que la Universidad Juárez AutónomadeTabascoasumesucompromisodedivulgación de la investigación y de promoción del pensamiento científico entre los jóvenes tabasqueños más allá de sus aulas. Entre las debilidades que se tiene en el presente, es la falta de profundidad en la información cualitativa, futuros estudios se pueden realizar abordando el alcance y la medición del fomento a las vocaciones científicas.

En ese sentido, los Clubes de Ciencia conformados y que han operado desde el 2014 hasta 2018, pueden considerarse un caso de éxito porque sus actividades han trascendido los muros de una institución, se ha trabajado en primera instancia para la Educación Media Superior, pero también en Educación Básica y Media, Instituciones de otros Estados, Congresos nacionales e internacionales, y sociedad en general, por lo que su cobertura no es sólo local. Con todas las acciones y actividades que se presentaron en este trabajo queda demostrado que los CUC son un manifiesto activo de la Responsabilidad Social que la Universidad ha adquirido.

\section{| Referencias}

Castells, M. (1999). La sociedad red. La era de la información: economía, sociedad y cultura, Volumen 1. (Trad. Carmen Martínez Gimeno). Siglo XXI.

Díaz, L., y Rojas, M. (2020). Elementos a considerar para el diseño de un Club de Ciencia y Tecnología como estrategia de proyección social desde el Departamento de Tecnología de la Universidad Pedagógica Nacional de Colombia. https://cutt.ly/IH6nmNu

Gaete Quezada, R. (2015). La responsabilidad Social Universitaria desde la perspectiva de las partes interesadas: un estudio de caso. Actualidades Investigativas en Educación, 15(1), 1-29. https://doi.org/gg7x38
López, J.C. (2013). La importancia de la educación científica. Cuadernos de Cultura Científica. https://cutt.ly/ mTegAR8

Lozada-Chávez, I. (2006). Divulgación Científica. https:// cutt.ly/iTegDzc

Mochan, L. (2020). La importancia de la cultura científica en el conocimiento de la sociedad mexicana. https://cutt.ly/ $\underline{n T e g F c N}$

Monreal Ávila, D. (2014). Gaceta del día jueves 13 de febrero de 2014; Gaceta LXII/2SPO-81/45698. Gaceta del Senado. Senado de la República Mexicana. https://cutt. ly/qTegGgg

Organización para la Cooperación y el Desarrollo Económicos [OCDE]. (2018a). Gross domestic spending on R\&D. https://cutt.ly/KTegHOr

Organización para la Cooperación y el Desarrollo Económicos [OCDE]. (2018b). Researchers. https://cutt.ly/ EH6muMD

Organización de las Naciones Unidas para la Educación, la Ciencia y la Cultura [UNESCO]. (2015). Science Report: towards 2030. https://cutt.ly/8H61d5H

Organización de las Naciones Unidas para la Educación, la Ciencia y la Cultura [UNESCO]. (2009). Conferencia Mundial sobre la Educación Superior 2009: La nueva dinámica de la Educación Superior y la investigación para el cambio social y el desarrollo [comunicado de prensa]. https://cutt.ly/eTeg3Tj

Organización de las Naciones Unidas para la Educación, la Ciencia y la Cultura [UNESCO]. (1984). Manual para el fomento de las actividades científicas y tecnológicas juveniles. https://cutt.ly/NH6NBwq

Ostrom, E. (1990). Governing the commons: The evolution of institutions for collective action. Cambridge university press. https://cutt.ly/WTtJTjU

Red Mexicana de Talleristas de Ciencia. (2021). Recreación en cadena. https://cutt.ly/PTegZOp

Remolina, G. (2003) La responsabilidad social de la universidad. Nómadas, 19, 239-246.

Ruiz Galicia, C.A. (2017). La importancia de la cultura científca en México. https://cutt.ly/mTegKP6 
Sagan, C. (2017). El mundo y sus demonios. La ciencia como una luz en la oscuridad. (Trad. Dolors Udina Abelló). Grupo Planeta (Obra original publicada en 1995).

Scheler, M. (1979). Los valores. Paidos.

Sociedad Mexicana para la Divulgación de la Ciencia y la Técnica [SOMEDICYT]. (2019). ¿Quiénes somos?. https://cutt.ly/gTehqYI

Suárez-Rodríguez, C., Léon-Lomelí, L., Gamboa-León, M. \& Zamora-Pedraza, C. (2021). Formación científica STEM, experiencias de aprendizaje a partir de clubes de ciencias. Rede Amazônica de Educação em Ciências e Matemática. 9(1). https://doi.org/hwp3

Universidad Juárez Autónoma de Tabasco [UJAT] (2012). Plan de Desarrollo Institucional 2012 - 2016. Editorial UJAT. https://cutt.ly/YTdsITl

Vallaeys, F.; De la Cruz, C. y Sasia P. M. (2009). Manual de primeros pasos en responsabilización social universitaria. Construyendo ciudadanía en universidades responsables. McGrawHill. https://cutt.ly/hTegN7R

Vallaeys, F. (sin fecha). ¿Qué es la Responsabilidad Social Universitaria?. https://cutt.ly/CTdsVbK

Vallaeys, F. (2014). La responsabilidad social universitaria: un nuevo modelo universitario contra la mercantilización. Revista Iberoamericana de Educación Superior, 5(12). https://cutt.ly/QTdsHx3

Yin, R.K. (1984/1989). Case Study Research: design and Methods, Applied social research. Methods Series. Sage. 